HETEROCYCLES, Vol. 88, No. 1, 2014, pp. 223 - 231. @ 2014 The Japan Institute of Heterocyclic Chemistry

Received, 13th April, 2013, Accepted, 17th June, 2013, Published online, 20th June, 2013

DOI: 10.3987/COM-13-S(S)5

\title{
PALLADIUM-CATALYZED TETRAARYLATION OF \\ 5,15-DIALKYLPORPHYRINS WITH ARYL BROMIDES
}

\section{Yutaro Yamamoto, Sumito Tokuji, Takayuki Tanaka, Hideki Yorimitsu, ${ }^{* \star}$ and} Atsuhiro Osuka*

Department of Chemistry, Graduate School of Science, Kyoto University, Sakyo-ku, Kyoto 606-8502, Japan; ACT-C, Japan Science and Technology Agency, Sakyo-ku, Kyoto 606-8502, Japan; E-mail: yori@kuchem.kyoto-u.ac.jp, osuka@kuchem.kyoto-u.ac.jp

\begin{abstract}
Nickel complexes of 5,15-dialkylporphyrins are subjected to palladium-catalyzed direct arylation under the modified Fagnou conditions. The arylation takes place still exclusively at the four less hindered $\beta$ positions although the meso-nonyl, hexyl, and propyl groups are considered to impose less steric hindrance than the meso-3,5-di-tert-butylphenyl group in the previous report.
\end{abstract}

\section{INTRODUCTION}

Due to the important roles that porphyrins adopt in a variety of pivotal biological processes, chemists have devoted much time to the design and synthesis of new artificial porphyrins that can be utilized in advanced functional materials. ${ }^{1}$ Peripheral functionalizations of a porphyrin core is an effective strategy that allows for the systematic construction of porphyrin-based architechtures. ${ }^{2}$ Palladium-catalyzed cross-coupling reactions ${ }^{3}$ can be used to successfully introduce a direct carbon-carbon bond at the periphery of a porphyrin. ${ }^{1,2,4}$ However, these reactions generally take place in moderate yield. Unlike benzene-based building blocks, which are cheap and readily available, prefunctionalized porphyrins such as bromoporphyrins and borylporphyrins are far more precious. It is therefore essential that extensive efforts be made to develop much more efficient, scalable, and reliable synthetic methodologies for achieving these highly desirable molecules.

A recent dramatic growth in reports of transition-metal-catalyzed direct $\mathrm{C}-\mathrm{H}$ arylations has changed the

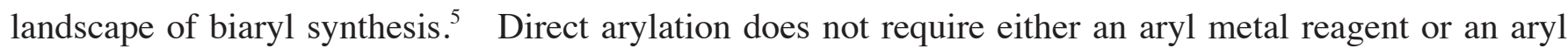
halide and therefore represents the ideal arylation. We have been interested in the modification of functional aromatics by direct arylation ${ }^{6,7}$ and we have recently developed conditions for palladium-

This paper is dedicated to Professor Victor Snieckus on the occasion of his 77th birthday. 
catalyzed $\beta$-selective direct arylation of porphyrins ${ }^{8,9}$ based on procedures originally reported by Fagnou. ${ }^{10,11}$ Our direct $\beta$-arylation has proved to be applicable to nickel complexes of 5,10,15-triarylporphyrin, 5,15-diarylporphyrin, and 5,10,15-triaryl-20-formylporphyrins bearing bulky 3,5-di-tert-butylphenyl groups as the aryl groups at their meso positions. Since the regioselective outcome of porphyrin arylation is heavily governed by sterics, the size of the group that is present at the meso position is a critical factor. To test this hypothesis, we have synthesized a small series of porphyrins bearing alkyl chains of various sizes at the meso positions. The regioselective outcome of direct arylation reactions of this small series of porphyrins is reported.

\section{RESULTS AND DISCUSSION}

Three dialkylporphyrins, $\mathbf{1 a}\left(\mathrm{R}=\mathrm{C}_{9} \mathrm{H}_{19}\right), \mathbf{1 b}\left(\mathrm{R}=\mathrm{C}_{6} \mathrm{H}_{13}\right)$, and $\mathbf{1 c}\left(\mathrm{R}=\mathrm{C}_{3} \mathrm{H}_{7}\right)$, were prepared and subjected to the palladium-catalyzed tetraarylation ${ }^{8}$ (Table 1 ). Tetraarylation proceeded very smoothly to afford the corresponding tetraarylated products $\mathbf{2}$ as the sole isolated products with exclusive regioselectivity. Considering the reaction involves the simultaneous formation of four $\mathrm{C}-\mathrm{C}$ bonds, the yields are extremely high. ${ }^{12}$ The presence of Davephos (2-dicyclohexylphosphino-2'-dimethylaminobiphenyl) led to slightly better yields of 2 (Entries 1, 3, 5, 7 vs. 2, 4, 6, 8). Arylation with bulky 2-bromotoluene also proceeded

Table 1. Direct arylation of 5,15-dialkylporphyrin nickel complexes 1
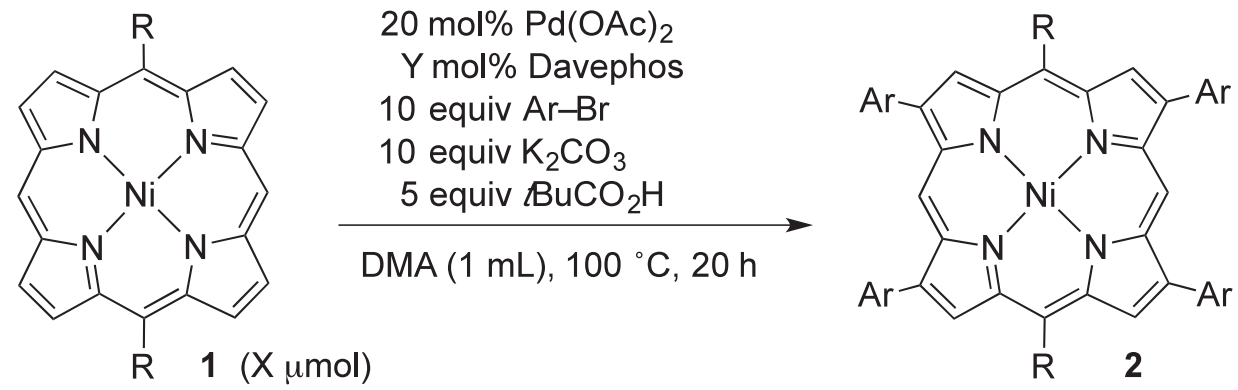

\begin{tabular}{lllllll}
\hline Entry & $\mathrm{R}$ & $\mathrm{X}$ & $\mathrm{Y}$ & $\mathrm{Ar}$ & $\mathbf{2}$ & Yield $/ \%$ \\
\hline 1 & $\mathrm{C}_{9} \mathrm{H}_{19}$ & 50 & 40 & 3,5-dimethylphenyl & $\mathbf{2 a}$ & 97 \\
2 & $\mathrm{C}_{9} \mathrm{H}_{19}$ & 50 & 0 & 3,5-dimethylphenyl & $\mathbf{2 a}$ & 85 \\
3 & $\mathrm{C}_{9} \mathrm{H}_{19}$ & 50 & 40 & 2-methylphenyl & $\mathbf{2 a}$ & 98 \\
4 & $\mathrm{C}_{9} \mathrm{H}_{19}$ & 50 & 0 & 2-methylphenyl & $\mathbf{2 a}$ & 83 \\
5 & $\mathrm{C}_{6} \mathrm{H}_{13}$ & 25 & 40 & 3,5-dimethylphenyl & $\mathbf{2 b}$ & 96 \\
6 & $\mathrm{C}_{6} \mathrm{H}_{13}$ & 25 & 0 & 3,5-dimethylphenyl & $\mathbf{2 b}$ & 78 \\
7 & $\mathrm{C}_{3} \mathrm{H}_{7}$ & 25 & 40 & 3,5 -dimethylphenyl & $\mathbf{2 c}$ & 77 \\
8 & $\mathrm{C}_{3} \mathrm{H}_{7}$ & 25 & 0 & 3,5 -dimethylphenyl & $\mathbf{2 c}$ & 76 \\
\hline
\end{tabular}


favorably (Entries 3,4). The reactivities of $\mathbf{1 a}$ and $\mathbf{1 b}$ are comparable to those of meso-diarylporphyrins. On the other hand, the yields of $\mathbf{2 c}$ were slightly lower (Entries 7,8 ). We speculate this is because 1c is equipped with short propyl groups that exhibit poor solubility in $N, N$-dimethylacetamide (DMA). Unfortunately, we could not obtain crystals of $\mathbf{2}$ suitable for X-ray crystallographic analysis. Nevertheless, we can safely assume that the four aryl groups are located at the less hindered $\beta$ positions $(2$, $8,12,18$ positions), as indicated. It is improbable for the four aryl groups to be introduced at the more crowded $\beta$ positions $(3,7,13,17$ positions) adjacent to the alkyl groups since Fagnou's direct arylation has been established to follow steric control. ${ }^{10}$ The anticipated regioselectivity is additionally confirmed by ${ }^{1} \mathrm{H}$ NMR spectroscopy (Table 2). The meso-H signal of porphyrin $\mathbf{2 a}$ bearing four 3,5-dimethylphenyl groups appear at $9.83 \mathrm{ppm}$ and is shifted downfield by $0.13 \mathrm{ppm}$ compared to that of starting material 1a $(9.70 \mathrm{ppm})$. In contrast, porphyrin $\mathbf{2} \mathbf{a}^{\prime}$ bearing four 2-methylphenyl groups shows its meso-H signal at $9.15 \mathrm{ppm}$, significantly shifted upfield by $0.55 \mathrm{ppm}$. This dramatic upfield shift most likely originates from the diatropic ring current of the $\beta$-benzene rings, which would be nearly perpendicular to the porphyrinic plane due to the steric crash with the ortho methyl group and would thus cover the meso protons. Notably, the arylation of 1a did not induce drastic changes in the chemical shifts of the signals corresponding to the methylene protons that are in closest proximity to the porphyrin core, as illustrated by limited upfield shifts, $0.14 \mathrm{ppm}$ in $\mathbf{2 a}$ and $0.07 \mathrm{ppm}$ in $\mathbf{2 a}$ '. The significant changes in the chemical shifts for the meso protons and the small changes for the methylene protons are strong evidence that the aryl groups introduced are located at the less hindered $\beta$ positions next to the unsubstituted meso positions. Furthermore, similar changes in the chemical shifts for meso protons were observed in the previous $\beta$-diarylation of 5,10,15-triarylporphyrin at the 2 and 18 positions, ${ }^{8}$ where the introduction of 3,5-dimethylphenyl groups and of 2-methylphenyl groups gave rise to a downfield shift (0.25 ppm) and an upfield shift (0.47 ppm), respectively.

The UV-visible absorption spectra of $\mathbf{1 a}, \mathbf{2 a}$, and $\mathbf{2} \mathbf{a}^{\prime}$ are illustrated in Figure 1. The introductions of the aryl groups in $\mathbf{2 a}$ and $\mathbf{2} \mathbf{a}^{\prime}$ obviously resulted in red-shifted absorptions. Notably, 2a bearing 3,5-dimethylphenyl groups shows more red-shifted absorption than 2a' bearing 2-methylphenyl groups. The difference in the absorption exhibits that $\mathbf{2} \mathbf{a}^{\prime}$ has less effective conjugation than $\mathbf{2} \mathbf{a}$ and implies that the 2-methylphenyl groups in $\mathbf{2} \mathbf{a}^{\prime}$ would be more tilted to the porphyrin plane than the 3,5-dimethylphenyl groups in 2a. This implication is consistent with the significant upfield shift of the meso protons in the ${ }^{1} \mathrm{H}$ NMR analysis of $\mathbf{2} \mathbf{a}^{\prime}$.

In conclusion, 5,15-dialkylporphyrin nickel complexes undergo palladium-catalyzed direct tetraarylation under modified Fagnou conditions using pivalic acid as a mediator. The arylation is high-yielding and takes place exclusively at the four less hindered $\beta$ positions as observed in the arylation of meso-3,5-di-tert-butylphenyl-substituted porphyrins. 
Table 2. Chemical shifts of notable protons

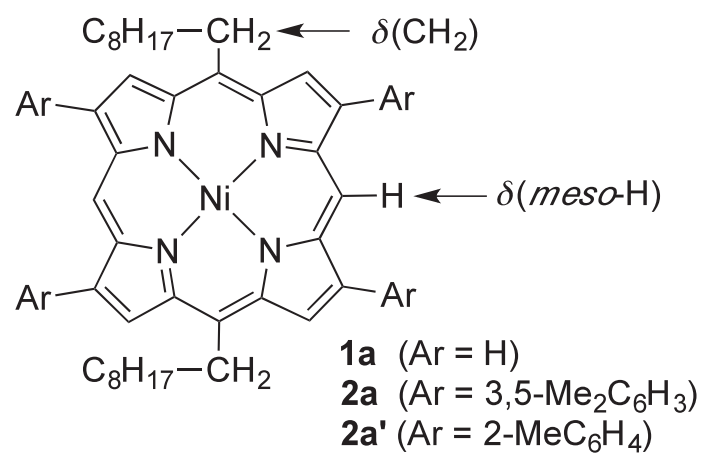

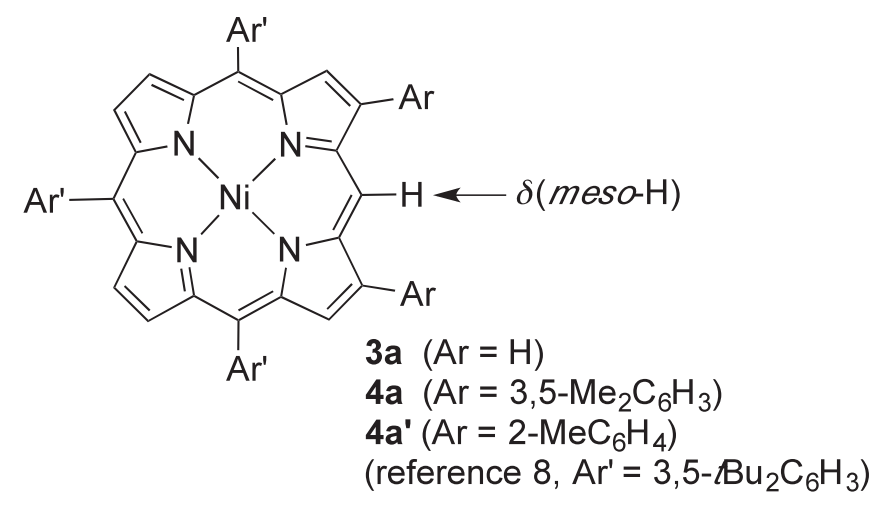

\begin{tabular}{lllll}
\hline Porphyrin & $\delta($ meso-H $) / \mathrm{ppm}$ & $\delta\left(\mathrm{CH}_{2}\right) / \mathrm{ppm}$ & $\Delta \delta(\text { meso- } \mathrm{H})^{\mathrm{a}} / \mathrm{ppm}$ & $\Delta \delta\left(\mathrm{CH}_{2}\right)^{\mathrm{a}} / \mathrm{ppm}$ \\
\hline $\mathbf{1 a}$ & 9.70 & 4.67 & - & - \\
$\mathbf{2 a}$ & 9.83 & 4.53 & +0.13 & -0.14 \\
$\mathbf{2 a}$ & 9.15 & 4.60 & -0.55 & -0.07 \\
$\mathbf{3 a}$ & 9.83 & - & - & - \\
$\mathbf{4 a}$ & 10.08 & - & +0.25 & - \\
$\mathbf{4 a}$ & 9.36 & - & -0.47 & - \\
\hline
\end{tabular}

${ }^{a}$ Difference of chemical shifts between the arylated product ( $\mathbf{2}$ or $\left.\mathbf{4}\right)$ and the parent porphyrin (1 or $\left.\mathbf{3}\right)$.

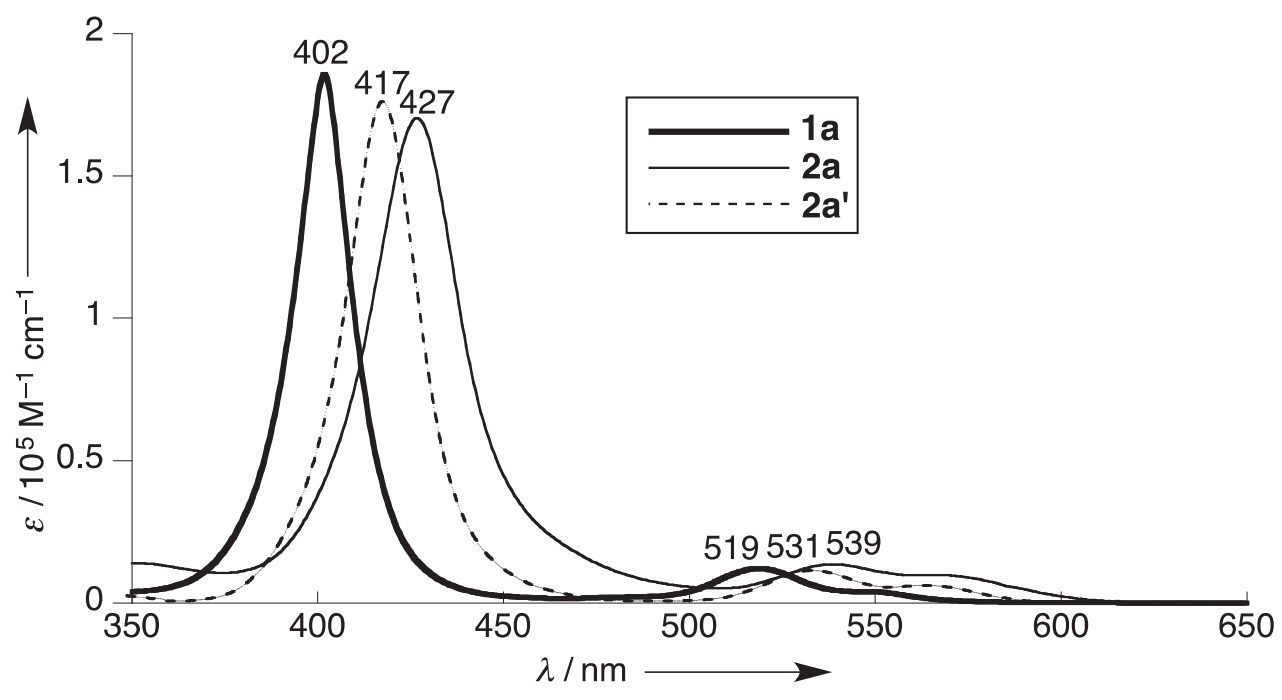

Figure 1. UV-visible absorption spectra of $\mathbf{1 a}, \mathbf{2 a}$, and $\mathbf{2} \mathbf{a}^{\prime}$

\section{EXPERIMENTAL}

${ }^{1} \mathrm{H}$ NMR $(600 \mathrm{MHz})$ and ${ }^{13} \mathrm{C}$ NMR $(151 \mathrm{MHz})$ spectra were taken on a JEOL ECA-600 spectrometer. Chemical shifts are reported on a delta scale in ppm relative to residual $\mathrm{CHCl}_{3}(\delta=7.26 \mathrm{ppm})$ for ${ }^{1} \mathrm{H}$ NMR and to $\mathrm{CDCl}_{3}(\delta=77.16 \mathrm{ppm})$ for ${ }^{13} \mathrm{C} \mathrm{NMR}$. Spectroscopic grade solvents were used for all 
spectroscopic studies without further purification. UV-Visible absorption spectra were recorded on a Shimadzu UV-2550 and Shimadzu UV-3600PC spectrometer. ESI-TOF-MS spectra were recorded on a Bruker Daltonics micrOTOF II instrument using a positive-ion mode. TLC analyses were performed on commercial glass plates bearing a $0.25-\mathrm{mm}$ layer of Merck Silica gel $60 \mathrm{~F}_{254}$. Preparative separations were performed by silica-gel column chromatography (Wako gel C-200, C-300, or C-400).

Unless otherwise noted, materials obtained from commercial suppliers were used without further purification. DMA was distilled from barium oxide and kept over Molecular Sieves $4 \AA$ under argon. Palladium acetate, nickel acetylacetonate, and Davephos were purchased from Wako Pure Chemicals, nacalai tesque, and Aldrich, respectively.

Starting Materials. Free base 5,15-dialkylporphyrins were prepared according to the literature. ${ }^{13}$ Subsequent nickelation proceeded quantitatively by treatment of the free base porphyrins with an excess amount of nickel acetylacetonate in boiling toluene overnight.

(5,15-Dinonylporphyrinato)nickel (1a): ${ }^{1} \mathrm{H} \mathrm{NMR}\left(600 \mathrm{MHz}, \mathrm{CDCl}_{3}\right) \delta=9.70(\mathrm{~s}, 2 \mathrm{H}$, meso), $9.43(\mathrm{~d}, 4 \mathrm{H}$, $J=4.8 \mathrm{~Hz}, \beta), 9.17(\mathrm{~d}, 4 \mathrm{H}, J=4.8 \mathrm{~Hz}, \beta), 4.67$ (t, 4H, $J=8.3 \mathrm{~Hz}$, nonyl), 2.36 (m, 4H, nonyl), 1.65 (m, 4H, nonyl), 1.46 (m, 4H, nonyl), 1.35-1.21 (m, 16H, nonyl), $0.86\left(\mathrm{t}, 6 \mathrm{H}, J=6.9 \mathrm{~Hz}\right.$, nonyl) ppm; ${ }^{13} \mathrm{C}$ $\operatorname{NMR}\left(151 \mathrm{MHz}, \mathrm{CDCl}_{3}\right): \delta=142.68,141.55,132.27,129.56,117.45,104.27,37.80,34.38,32.04,30.62$, 29.76 (overlap), 29.50, 22.81, 14.25 ppm; UV-vis $\left(\mathrm{CH}_{2} \mathrm{Cl}_{2}\right): \lambda_{\max }\left(\varepsilon\left[\mathrm{M}^{-1} \mathrm{~cm}^{-1}\right]\right)=402(190000), 519 \mathrm{~nm}$ (12000); MS (ESI, positive): $m / z=619.3279$. Calcd for $\mathrm{C}_{38} \mathrm{H}_{49} \mathrm{~N}_{4} \mathrm{Ni}: 619.3305[M+\mathrm{H}]^{+}$.

(5,15-Dihexylporphyrinato)nickel (1b): ${ }^{1} \mathrm{H} \mathrm{NMR}\left(600 \mathrm{MHz}, \mathrm{CDCl}_{3}\right) \delta=9.71(\mathrm{~s}, 2 \mathrm{H}$, meso $), 9.45(\mathrm{~d}, 4 \mathrm{H}$, $J=4.6 \mathrm{~Hz}, \beta), 9.18(\mathrm{~d}, 4 \mathrm{H}, J=4.6 \mathrm{~Hz}, \beta), 4.68$ (t, 4H, $J=8.3 \mathrm{~Hz}$, hexyl), 2.37 (m, 4H, hexyl), 1.66 (m, 4H, hexyl), 1.45 (m, 4H, hexyl), 1.35 (m, 4H, hexyl), 0.91 (t, 6H, $J=7.8 \mathrm{~Hz}$, hexyl) ppm; ${ }^{13} \mathrm{C}$ NMR $(151$ $\left.\mathrm{MHz}, \mathrm{CDCl}_{3}\right): \delta=142.65,141.52,132.23,129.50,117.40,104.01,37.77,34.36,31.96,30.29,22.85$, 14.29 ppm; UV-vis $\left(\mathrm{CH}_{2} \mathrm{Cl}_{2}\right): \lambda_{\max }\left(\varepsilon\left[\mathrm{M}^{-1} \mathrm{~cm}^{-1}\right]\right)=402$ (210000), 518 (13000), $550 \mathrm{~nm}$ (4000); MS (ESI, positive): $m / z=535.2345$. Calcd for $\mathrm{C}_{32} \mathrm{H}_{37} \mathrm{~N}_{4} \mathrm{Ni}: 535.2366[M+\mathrm{H}]^{+}$.

(5,15-Dipropylporphyrinato)nickel (1c): ${ }^{1} \mathrm{H} \mathrm{NMR}\left(600 \mathrm{MHz}, \mathrm{CDCl}_{3}\right) \delta=9.70$ (s, 2H, meso), 9.44 (d, $4 \mathrm{H}, J=4.8 \mathrm{~Hz}, \beta), 9.17(\mathrm{~d}, 4 \mathrm{H}, J=4.8 \mathrm{~Hz}, \beta), 4.66$ (t, 4H, $J=7.8 \mathrm{~Hz}$, propyl), 2.40 (m, 4H, propyl), 1.21 (t, $6 \mathrm{H}, J=7.3 \mathrm{~Hz}$, propyl) ppm; ${ }^{13} \mathrm{C}$ NMR $\left(151 \mathrm{MHz}, \mathrm{CDCl}_{3}\right): \delta=142.81,141.62,132.35,129.71,117.22$, 104.08, 36.34, 30.69, 15.03 ppm; UV-vis $\left(\mathrm{CH}_{2} \mathrm{Cl}_{2}\right): \lambda_{\max }\left(\varepsilon\left[\mathrm{M}^{-1} \mathrm{~cm}^{-1}\right]\right)=401$ (210000), 518 (14000), 550 $\mathrm{nm}$ (5000); MS (ESI, positive): $m / z=451.1419$. Calcd for $\mathrm{C}_{26} \mathrm{H}_{25} \mathrm{~N}_{4} \mathrm{Ni}: 451.1427[M+\mathrm{H}]^{+}$.

Direct Arylation of 5,15-Dinonylporphyrin: 5,15-Dinonylporphyrin 1a $(0.050 \mathrm{mmol}, 31.1 \mathrm{mg})$, $\mathrm{Pd}(\mathrm{OAc})_{2}(0.010 \mathrm{mmol}, 2.2 \mathrm{mg})$, Davephos (0.020 mmol, $\left.7.9 \mathrm{mg}\right), \mathrm{K}_{2} \mathrm{CO}_{3}(0.50 \mathrm{mmol}, 69.1 \mathrm{mg})$, and $t \mathrm{BuCO}_{2} \mathrm{H}(0.25 \mathrm{mmol}, 25.6 \mathrm{mg})$ were added to a reaction flask. The reaction flask was purged with argon, and DMA (1.0 mL) and aryl bromide $(0.50 \mathrm{mmol}, 70 \mu \mathrm{L}$ for 1-bromo-3,5-dimethylbenzene or 60 $\mu \mathrm{L}$ for 2-bromotoluene) were added. The reaction mixture was then stirred at $100{ }^{\circ} \mathrm{C}$ for $20 \mathrm{~h}$. The 
resulting mixture was allowed to cool to room temperature, diluted with $\mathrm{CH}_{2} \mathrm{Cl}_{2}$, and passed through a pad of Celite with copious washings with $\mathrm{CH}_{2} \mathrm{Cl}_{2}$. After evaporation of the solvent, the product was separated by silica-gel column chromatography $\left(\mathrm{CH}_{2} \mathrm{Cl}_{2} /\right.$ hexane $)$. Further purification by recrystallization from $\mathrm{CH}_{2} \mathrm{Cl}_{2} / \mathrm{MeOH}$ yielded the corresponding product (2a, $50.2 \mathrm{mg}, 0.0484 \mathrm{mmol}$, 97\%; 2a' , $48.0 \mathrm{mg}, 0.0490 \mathrm{mmol}, 98 \%$ ).

Direct Arylation of 5,15-Dihexyl- or 5,15-Dipropylporphyrin: 5,15-Dihexylporphyrin 1 b (0.025 mmol, $13.4 \mathrm{mg})$ or 5,15-dipropylporphyrin 1c $(0.025 \mathrm{mmol}, 11.3 \mathrm{mg}), \mathrm{Pd}(\mathrm{OAc})_{2}(0.005 \mathrm{mmol}, 1.1 \mathrm{mg})$, Davephos (0.010 mmol, $3.9 \mathrm{mg}), \mathrm{K}_{2} \mathrm{CO}_{3}(0.25 \mathrm{mmol}, 34.5 \mathrm{mg})$, and $t \mathrm{BuCO}_{2} \mathrm{H}(0.125 \mathrm{mmol}, 12.8 \mathrm{mg})$ were placed in a reaction flask under argon. DMA $(1.0 \mathrm{~mL})$ and 1-bromo-3,5-dimethylbenzene $(0.25$ mmol, $35 \mu \mathrm{L}$ ) were added, and the whole mixture was heated at $100{ }^{\circ} \mathrm{C}$ for $20 \mathrm{~h}$. The resulting mixture was allowed to cool to ambient temperature, diluted with $\mathrm{CH}_{2} \mathrm{Cl}_{2}$, and filtered through a pad of Celite with copious washings with $\mathrm{CH}_{2} \mathrm{Cl}_{2}$. The filtrate was concentrated in vacuo. Chromatographic purification on silica gel $\left(\mathrm{CH}_{2} \mathrm{Cl}_{2}\right.$ /hexane) followed by recrystallization from $\mathrm{CH}_{2} \mathrm{Cl}_{2} / \mathrm{MeOH}$ afforded $2 \mathbf{b}$ (22.8 mg, $0.0240 \mathrm{mmol}, 96 \%)$ or $2 \mathrm{c}(16.6 \mathrm{mg}, 0.0191 \mathrm{mmol}, 77 \%)$.

[2,8,12,18-Tetrakis(3,5-dimethylphenyl)-5,15-dinonylporphyrinato]nickel (2a): ${ }^{1} \mathrm{H}$ NMR (600 MHz, $\left.\mathrm{CDCl}_{3}\right) \delta=9.83(\mathrm{~s}, 2 \mathrm{H}$, meso), $9.27(\mathrm{~s}, 4 \mathrm{H}, \beta), 7.68(\mathrm{~s}, 8 \mathrm{H}, \mathrm{Ar}-o), 7.20(\mathrm{~s}, 4 \mathrm{H}, \mathrm{Ar}-p), 4.53(\mathrm{t}, 4 \mathrm{H}, J=8.7$ $\mathrm{Hz}$, nonyl), 2.53 (s, 24H, Ar-Me), 2.34 (m, 4H, nonyl), 1.60 (m, 4H, nonyl), 1.42 (m, 4H, nonyl), $1.32-1.18\left(\mathrm{~m}, 16 \mathrm{H}\right.$, nonyl), $0.84\left(\mathrm{t}, 6 \mathrm{H}, J=6.9 \mathrm{~Hz}\right.$, nonyl) ppm; ${ }^{13} \mathrm{C} \mathrm{NMR}\left(151 \mathrm{MHz}, \mathrm{CDCl}_{3}\right): \delta=146.29$, 140.93 , 139.73 , 138.51 , 136.42, 129.24, 128.74, 127.30, 116.55, 103.95, 37.35, 34.05, 32.02, 30.54, 29.76, 29.71, 29.51, 22.79, 21.73, 14.23 ppm; UV-vis $\left(\mathrm{CH}_{2} \mathrm{Cl}_{2}\right): \lambda_{\max }\left(\varepsilon\left[\mathrm{M}^{-1} \mathrm{~cm}^{-1}\right]\right)=427$ (170000), 539 (15000), $565 \mathrm{~nm}$ (10000); MS (ESI-MS, positive): $\mathrm{m} / z=1057.5657$. Calcd for $\mathrm{C}_{70} \mathrm{H}_{80} \mathrm{~N}_{4} \mathrm{NiNa}: 1057.5629$ $[M+\mathrm{Na}]^{+}$.

[2,8,12,18-Tetrakis(2-methylphenyl)-5,15-dinonylporphyrinato]nickel (2a'): ${ }^{1} \mathrm{H}$ NMR (600 $\mathrm{MHz}$, $\left.\mathrm{CDCl}_{3}\right): \delta=9.30(\mathrm{~s}, 4 \mathrm{H}, \beta), 9.15$ (s, 2H, meso), $7.76(\mathrm{~d}, 4 \mathrm{H}, J=5.8 \mathrm{~Hz}, \operatorname{Ar}-o), 7.49-7.41$ (m, 12H, Ar- $m$ Ar-p), 4.60 (t, 4H, $J=8.0 \mathrm{~Hz}$, nonyl), 2.43 (s, 12H, Ar-Me), 2.38 (m, 4H, nonyl), 1.60 (m, 4H, nonyl), 1.42 (m, 4H, nonyl), 1.30-1.18 (m, 16H, nonyl), 0.83 (t, 6H, $J=7.1 \mathrm{~Hz}$, nonyl) ppm; ${ }^{13} \mathrm{C} \mathrm{NMR}(151 \mathrm{MHz}$, $\left.\mathrm{CDCl}_{3}\right): \delta=145.87,141.38,140.97,137.56,135.77,132.52,130.61,129.00,128.16,125.70,117.27$, $103.18,37.76,34.32,32.02,30.60,29.76,29.47,22.78,21.37,20.89,14.23$ ppm; UV-vis $\left(\mathrm{CH}_{2} \mathrm{Cl}_{2}\right): \lambda_{\max }(\varepsilon$ $\left.\left[\mathrm{M}^{-1} \mathrm{~cm}^{-1}\right]\right)=417(180000), 531(11000), 561 \mathrm{~nm}(6000)$; MS (ESI-MS, positive): $\mathrm{m} / z=979.5140$. Calcd for $\mathrm{C}_{66} \mathrm{H}_{73} \mathrm{~N}_{4} \mathrm{Ni}: 979.5183[M+\mathrm{H}]^{+}$.

[2,8,12,18-Tetrakis(3,5-dimethylphenyl)-5,15-dihexylporphyrinato]nickel (2b): ${ }^{1} \mathrm{H}$ NMR (600 MHz, $\left.\mathrm{CDCl}_{3}\right) \delta=9.83$ (s, 2H, meso), 9.28 (s, 4H, $\beta$ ), 7.68 (s, 8H, Ar-o), 7.20 (s, 4H, Ar-p), 4.53 (t, 4H, $J=8.0$ $\mathrm{Hz}$, hexyl), 2.53 (s, 24H, Ar-Me), 2.34 (m, 4H, hexyl), 1.61 (m, 4H, hexyl), 1.41 (m, 4H, hexyl), 1.33 (m, 4H, hexyl), 0.89 (t, $6 \mathrm{H}, J=7.3 \mathrm{~Hz}$, hexyl) ppm; ${ }^{13} \mathrm{C} \mathrm{NMR}\left(151 \mathrm{MHz}, \mathrm{CDCl}_{3}\right): \delta=146.38,140.98,139.80$, 
$138.54,136.44,129.28,128.77,127.35,116.63,103.96,37.45,34.16,31.96,30.30,22.88,21.74,14.30$ ppm; UV-vis $\left(\mathrm{CH}_{2} \mathrm{Cl}_{2}\right): \lambda_{\max }\left(\varepsilon\left[\mathrm{M}^{-1} \mathrm{~cm}^{-1}\right]\right)=426$ (160000), 538 (12000), $568 \mathrm{~nm}$ (8000); MS (ESI-MS, positive): $m / z=951.4823$. Calcd for $\mathrm{C}_{64} \mathrm{H}_{69} \mathrm{~N}_{4} \mathrm{Ni}: 951.4870[M+\mathrm{H}]^{+}$.

[2,8,12,18-Tetrakis(3,5-dimethylphenyl)-5,15-dipropylporphyrinato]nickel (2c): ${ }^{1} \mathrm{H}$ NMR (600 MHz, $\left.\mathrm{CDCl}_{3}\right) \delta=9.87(\mathrm{~s}, 2 \mathrm{H}$, meso), $9.32(\mathrm{~s}, 4 \mathrm{H}, \beta), 7.70$ (s, 8H, Ar-o), 7.20 (s, 4H, Ar-p), 4.56 (t, 4H, $J=8.0$ $\mathrm{Hz}$, propyl), 2.54 (s, 24H, Ar-Me), 2.39 (m, 4H, propyl), 1.20 (t, 6H, $J=7.4 \mathrm{~Hz}$, propyl) ppm; ${ }^{13} \mathrm{C}$ NMR $\left(151 \mathrm{MHz}, \mathrm{CDCl}_{3}\right): \delta=146.38,140.01,139.80,138.52,136.40,129.27,128.75,127.38,116.37,104.00$, 36.13, 34.16, 30.42, 21.73, 15.08 ppm; UV-vis $\left(\mathrm{CH}_{2} \mathrm{Cl}_{2}\right): \lambda_{\max }\left(\varepsilon\left[\mathrm{M}^{-1} \mathrm{~cm}^{-1}\right]\right)=426$ (190000), 537 (15000), $568 \mathrm{~nm}$ (10000); MS (ESI-MS, positive): $m / z=867.3888$. Calcd for $\mathrm{C}_{58} \mathrm{H}_{57} \mathrm{~N}_{4} \mathrm{Ni}: 867.3931[M+\mathrm{H}]^{+}$.

\section{ACKNOWLEDGEMENTS}

This work was supported by Grants-in-Aid (nos. 22245006 (A), 20108006 “pi-Space”, 24685007, and 22406721 "Reaction Integration") from MEXT. T.T. and S.T. acknowledge JSPS Fellowship for Young Scientists. H.Y. thanks financial support from Kinki Invention Center.

\section{REFERENCES}

1. 'Handbook of Porphyrin Science,' Vol. 1-10, ed. by K. M. Kadish, K. M. Smith, and R. Guilard, World Scientific Publishing, Singapore, 2010; 'Handbook of Porphyrin Science,' Vol. 11-15, ed. by K. M. Kadish, K. M. Smith, and R. Guilard, World Scientific Publishing, Singapore, 2011; 'Handbook of Porphyrin Science,' Vol. 16-25, ed. by K. M. Kadish, K. M. Smith, and R. Guilard, World Scientific Publishing, Singapore, 2012; 'The Porphyrin Handbook,' Vol. 1-10, ed. by K. M. Kadish, K. M. Smith, and R. Guilard, Academic Press, San Diego, 2000; 'Handbook of Porphyrin Science,' Vol. 11-20, ed. by K. M. Kadish, K. M. Smith, and R. Guilard, Academic Press, San Diego, 2003; D. Dolphin, 'The Porphyrins,' Vol. 1 and 2, Academic Press, New York, 1979.

2. Recent reviews: M. O. Senge, Chem. Commun., 2011, 47, 1943; S. Horn, K. Dahms, and M. O. Senge, J. Porphyrins Phthalocyanines, 2008, 12, 1053; M. O. Senge, Acc. Chem. Res., 2005, 38, 733; H. Shinokubo and A. Osuka, Chem. Commun., 2009, 1011; T. Ren, Chem. Rev., 2008, 108, 4185; B. M. J. M. Suijkerbuijk and R. J. M. Klein Gebbink, Angew. Chem. Int. Ed., 2008, 47, 7396; F. Atefi and D. P. Arnold, J. Porphyrins Phthalocyanines, 2008, 12, 801; M. G. H. Vicente and K. M. Smith, J. Porphyrins Phthalocyanines, 2004, 8, 26; W. M. Sharman and J. E. van Lier, J. Porphyrins Phthalocyanines, 2000, 4, 441; J. Setsune, J. Porphyrins Phthalocyanines, 2004, 8, 93; S. Fox and R. W. Boyle, Tetrahedron, 2006, 62, 10039; A. M. V. M. Pereira, S. Richeter, C. Jeandon, J.-P. Gisselbrecht, J. Wytko, and R. Ruppert, J. Porphyrins Phthalocyanines, 2012, 16, 464; A. Satake, J. Synth. Org. Chem. Jpn., 2007, 65, 298; S. Hiroto, S. Yamaguchi, H. Shinokubo, and A. Osuka, J. 
Synth.Org. Chem.Jpn., 2009, 67, 688.

3. For general overview of cross-coupling reactions: 'Cross-Coupling Reactions: A Practical Guide,' ed. by N. Miyaura, Springer, Heidelberg, 2010; 'Metal-Catalyzed Cross-Coupling Reactions,' ed. by A. de Meijere and F. Diederich, Wiley, Weinheim, 2004; A. Suzuki and Y. Yamamoto, Chem. Lett., 2011, 40, 894; N. Miyaura, Bull. Chem. Soc.Jpn., 2008, 81, 1535; E. Negishi, Bull.Chem. Soc.Jpn., 2007, 80, 233; C. C. C. J. Seechurn, M. O. Kitching, T. J. Colacot, and V. Snieckus, Angew. Chem. Int. Ed., 2012, 51, 5062; N. Miyaura and A. Suzuki, Chem. Rev., 1995, 95, 2457; A. Suzuki, Angew. Chem. Int. Ed., 2011, 50, 6722; E. Negishi, Angew. Chem. Int. Ed., 2011, 50, 6738.

4. For pioneering works: S. G. DiMagno, V. S.-Y. Lin, and M. J. Therien, J. Am. Chem. Soc., 1993, 115, 2513; S. G. DiMagno, V. S.-Y. Lin, and M. J. Therien, J. Org. Chem., 1993, 58, 5983; V. S.-Y. Lin, S. G. DiMagno, and M. J. Therien, Science, 1994, 264, 1105; D. P. Arnold and L. J. Nitschinsk, Tetrahedron Lett., 1993, 34, 693; R. W. Wagner, T. E. Johnson, F. Li, and J. S. Lindsey, J. Org. Chem., 1995, 60, 5266; N. Aratani and A. Osuka, Org. Lett., 2001, 3, 4213; R. Gauler and N. Risch, Eur. J. Org. Chem., 1998, 1193; M. M. Khan, H. Ali, and J. E. van Lier, Tetrahedron Lett., 2001, 42, 1615; K. S. Chan, X. Zhou, B.-S. Luo, and T. C. W. Mak, J. Chem. Soc., Chem. Commun., 1994, 271; X. Zhou, Z.-y. Zhou, T. C. W. Mak, and K. S. Chan, J. Chem. Soc., Perkin Trans. 1, 1994, 2519; X. Zhou and K. S. Chan, J. Chem. Soc., Chem. Commun., 1994, 2493; A. G. Hyslop, M. A. Kellett, P. M. Iovine, and M. J. Therien, J. Am. Chem. Soc., 1998, 120, 12676; H. Hata, H. Shinokubo, and A. Osuka, J. Am. Chem. Soc., 2006, 128, 4119.

5. Very recent representative reviews: D. Alberico, M. E. Scott, and M. Lautens, Chem. Rev., 2007, 107, 174; T. Satoh and M. Miura, Chem. Lett., 2007, 36, 200; A. Mori and A. Sugie, Bull. Chem. Soc. Jpn., 2008, 81, 548; L. Ackermann, R. Vicente, and A. R. Kapdi, Angew. Chem. Int. Ed., 2009, 48, 9792; D. Lapointe and K. Fagnou, Chem. Lett., 2010, 39, 1118; K. Hirano and M. Miura, Synlett, 2011, 294; I. V. Seregin and V. Gevorgyan, Chem. Soc. Rev., 2007, 36, 1173; J. Wencel-Delord, T. Droge, F. Liu, and F. Glorius, Chem. Soc. Rev., 2011, 40, 4740; L. Ackermann, Chem. Rev., 2011, 111, 1315; C. S. Yeung and V. M. Dong, Chem. Rev., 2011, 111, 1215; T. Satoh and M. Miura, Synthesis, 2010, 3395; T. W. Lyons and M. S. Sanford, Chem. Rev., 2010, 110, 1147; C.-L. Sun, B.-J. Li, and Z.-J. Shi, Chem. Commun., 2010, 46, 677; X. Chen, K. M. Engle, D.-H. Wang, and J.-Q. Yu, Angew. Chem. Int. Ed., 2009, 48, 5094; G. P. McGlacken and L. M. Bateman, Chem. Soc. Rev., 2009, 38, 2447; F. Kakiuchi and T. Kochi, Synthesis, 2008, 3013; Y. J. Park, J.-W. Park, and C.-H. Jun, Acc. Chem. Res., 2008, 41, 222; E. M. Beccalli, G. Broggini, M. Martinelli, and S. Sottocornola, Chem. Rev., 2007, 107, 5318; J. Yamaguchi, A. D. Yamaguchi, and K. Itami, Angew. Chem. Int. Ed., 2012, 51, 8960; M. N. Hopkinson, J. Wencel-Delord, and F. Glorius, Angew. Chem. Int. Ed., 2012, 51, 10236; J. J. Mousseau and A. B. Charette, Acc. Chem. Res., 2013, 46, 412. 
6. S. Tokuji, T. Yurino, N. Aratani, H. Shinokubo, and A. Osuka, Chem. Eur. J., 2009, 15, 12208; Y. Mitamura, H. Yorimitsu, K. Oshima, and A. Osuka, Chem. Sci., 2011, 2, 2017; M. Iwasaki, H. Yorimitsu, and K. Oshima, Chem. Asian J., 2007, 2, 1430; S. Nakazono, S. Easwaramoorthi, D. Kim, H. Shinokubo, and A. Osuka, Org. Lett., 2009, 11, 5426; K. Osawa, N. Aratani, and A. Osuka, Tetrahedron Lett., 2009, 50, 3333; R. Ueno, D. Fujino, H. Yorimitsu, and A. Osuka, Chem. Eur. J., 2013, 19, in press, DOI: 10.1002/chem.201300623.

7. Review: H. Yorimitsu and A. Osuka, Asian J. Org. Chem., 2013, 2, in press, DOI: 10.1002/ajoc.201200183.

8. Y. Kawamata, S. Tokuji, H. Yorimitsu, and A. Osuka, Angew. Chem. Int. Ed., 2011, 50, 8867; S. Tokuji, H. Awane, H. Yorimitsu, and A. Osuka, Chem. Eur. J., 2013, 19, 64; Y. Yamamoto, S. Tokuji, T. Tanaka, H. Yorimitsu, and A. Osuka, Asian J. Org. Chem., 2013, 2, 320.

9. Inefficient intramolecular direct $\beta-\mathrm{C}-\mathrm{H}$ arylation of meso-haloaryl-substituted porphyrins by other groups: S. Fox and R. W. Boyle, Chem. Commun., 2004, 1322; A. N. Cammidge, P. J. Scaife, G. Berber, and D. L. Hughes, Org. Lett., 2005, 7, 3413.

10. M. Lafrance and K. Fagnou, J. Am. Chem. Soc., 2006, 128, 16496; S. I. Gorelsky, D. Lapointe, and K. Fagnou, J. Am. Chem. Soc., 2008, 130, 1084; H.-Y. Sun, S. I. Gorelsky, D. R. Stuart, L.-C. Campeau, and K. Fagnou, J. Org. Chem., 2010, 75, 8180; D. Lapointe, T. Markiewicz, C. J. Whipp, A. Toderian, and K. Fagnou, J. Org. Chem., 2011, 76, 749; S. I. Gorelsky, D. Lapointe, and K. Fagnou, J. Org. Chem., 2012, 77, 658 .

11. Related mechanistic work: M. Livendahl and A. M. Echavarren, Israel J. Chem., 2010, 50, 630; D. García-Cuadrado, A. A. C. Braga, F. Maseras, and A. M. Echavarren, J. Am. Chem. Soc., 2006, 128, 1066; D. García-Cuadrado, P. de Mendoza, A. A. C. Braga, F. Maseras, and A. M. Echavarren, J. Am. Chem. Soc., 2006, 128, 6880.

12. Reviews on the importance of one-pot multiple bond formation: S. Suga, D. Yamada, and J. Yoshida, Chem. Lett., 2010, 39, 404 and references cited therein; A. J. Bard, 'Integrated Chemical Systems,' Wiley, New York, 1994; 'Multicomponent Reactions,' ed. by J. Zhu and H. Bienaymé, Wiley, Weinheim, 2005.

13. Y. Nakamura, S. Y. Jang, T. Tanaka, N. Aratani, J. M. Lim, K. S. Kim, D. Kim, and A. Osuka, Chem. Eur. J., 2008, 14, 8279. 